\title{
Factores socio-demográficos, económicos y deportivos relacionados con la par- ticipación del equipo olímpico español en los JJ.00. de Pekín 2008 a Rio 2016 Socio-demographic, economic and sports factors related to the participation of the Spanish Olympic team in the Olympic Games from Beijing 2008 to Rio 2016 \\ *Alejandro Leiva-Arcas, *Raquel Vaquero-Cristóbal, *Antonio Sánchez-Pato, *Lucía Abenza-Cano, **María José Martínez-Patiño \\ *U niversidad Católica San Antonio de M urcia (España), **U niversidad deVigo (España)
}

\begin{abstract}
Resumen. El objetivo del presente estudio fue analizar los factores socio-demográficos, económicos y deportivos relaciona dos con los resultados de participación del equipo olímpico español en los JJ.00. de Pekín 2008, Londres 2012 y Rio de Janeiro 2016, analizando si el sexo del deportista provoca variaciones en el modelo. Se obtuvieron datos de 875 deportistas olímpicos pertenecientes al equipo español entre los años 2005 y 2016, así como de diferentes parámetros socio-demográ ficos, económicos, y deportivos. Se realizó un análisis de componentes principales que mostró que la población, el presupuesto deportivo, las licencias deportivas, los deportistas de alto nivel (DAN), los deportistas becados, la brecha de género y el PIB explicaban un 79,81\% de la varianza total. Al real izar el modelo en función del sexo se encontró un modelo similar al general en mujeres y hombres. En conclusión, la participación de hombres y mujeres en el olimpismo español se debe a una gran amalgama de variables, no existiendo grandes diferencias en la influencia de las mismas en función sexo.
\end{abstract}

Palabras clave: Deporte; Economía; Juegos O límpicos; O limpismo; Sociedad.

\begin{abstract}
The aim of this study was to analyze the socio-demographic, economic and sports factors related to the results of the Spanish O lympic team's participation in the O lympic Games of Beijing 2008, London 2012 and Rio de Janeiro 2016, analyzing whether the sex of the athlete causes variations in the model. Data was obtained from 8750 lympic athletes belonging to the Spanish team between 2005 and 2016, as well as from different socio-demographic, economic and sports parameters. A main component analysis was carried out which showed that the population, sportsbudget, sports licenses, top level athletes (TLA), scholarship awarded athletes, gender gap and GDP explained $79.81 \%$ of the total variance. When the gender model was carried out, a similar pattern to the general one was found in women and men. In conclusion, the participation of men and women in the Spanish Olympics is due to a great amalgam of variables, and there are no great differences in the influence of these variables according to gender.
\end{abstract}

Key Words: Sport; Economy; O lympic Games; O lympism; Society.

\section{Introducción}

Los J uegos 0 límpicos (JJ.00.) se han convertido en uno de los eventos deportivos más importantes del mundo, y uno de los acontecimientos transculturales que más atención despierta entre la población de todos los continentes (Curi, Knijnik, \& M ascarenhas, 2011). Su impacto, como fenómeno cultural, es visible en todos los ámbitos sociales (Perrino \& Vicente, 2018). A pesar de su corta duración, implican una larga preparación y sacrifico de sus participantes (Silva, Ribeiro, Silvestre, $\&$ Salerno, 2020). Tanto es así que, en cada país, los deportistas seleccionados para asistir a unos JJ.00. se convierten en celebridades, y los ganadores, se transforman en verdaderos héroes nacionales y referentes

Fecha recepción: 29-10-20. Fecha de aceptación: 12-01-21

Raquel Vaquero-Cristóbal

rvaquero@ucam.edu para la juventud (Garcia \& Miah, 2012).

Con el fin de conseguir el éxito en estetipo de eventos deportivos, el deporte de alto nivel se ha considerado de interés prioritario para numerosos países, incluyendo al Estado español. Fruto de ello, previo a los JJ.0 0. de Barcelona, se publicó la Ley 10/ 1990 del Deporte, la cual pretendía promover el desarrollo del deporte de alto nivel y al to rendimiento estableciendo para ello, entre otras medidas, un aporte de inversión pública a través de partidas anuales que son incluidas en los Presupuestos $G$ enerales del Estado, y que se siguen manteniendo hasta la actual idad. Con la llegada de esta Ley, en España se han ido estableciendo las líneas maestras para que los deportistas de alto nivel y alto rendimiento tengan los medios humanos y materiales suficientes y adecuados para su preparación (M oscoso, Fernández \& Rodríguez, 2014). 0 tro de los aspectos claves en el pla no social y donde se han aunado los esfuerzos es en la incorporación de estos deportistas al sistema educativo 
y su integración en el sistema social y laboral, pensando sobre todo en su inserción laboral una vez concluye su vida deportiva (Sánchez, Isidori, Arias \& Bada, 2018). Con tal fin, se publicó el Real Decreto 971/ 2007 de 13 de julio, donde se concretan las becas 0 ayudas que otorga el Consejo Superior de Deportes (CSD) para residir en los Centros de Alto Rendimiento (CAR), completar la formación académica y profesional, así como otras medidas tales como la reserva de plazas universitarias en estudios superiores a deportistas de élite.

Fruto de todo esto, en las últimas décadas ha habido una progresiva profesionalización, tecnificación y dependencia económica en el mundo del deporte, que afecta especialmente al deporte de élite y de alto rendimiento. Sin embargo, tras la década de los 90 y el llamado «periodo del Milagro Deportivo Español», la evolución ascendente de los éxitos se ha visto sensiblemente enlentecida (Leiva y Sánchez, 2019), fruto en parte a que muchos países han seguido un modelo similar de apoyo gubernamental al deporte olímpico (Green, 2007) así como por el avance de los medios tecnológicos de soporte al deportista, que generan una necesidad de continua innovación y cambio para conseguir el éxito (Haake, 2009). En este marco, en los últimos años se ha señalado la necesidad de construir model os vigentes que permitan indagar e interpretar, de forma cuantitativa, los parámetros que permiten a un país al canzar el éxito deportivo internacional (De Bosscher, De Knop, van Bottenburg, Shibli \& Bingham, 2009). La clave para un deporte de alto nivel potente, exitoso y de referencia internacional, pasa por disponer de un sistema deportivo sólido y bien diseñado en el que el apoyo a las élites deportivas sea el eje central del proyecto (Hoffman, Ging \& Ramasamy, 2002).

Si bien existen estudios relevantes en el ámbito internacional que tratan de explicar los factores que afectan al número de participantes y resultados obtenidos por un país en los JJ.00., desde enfoques socioeconómicos, políticoso incluso culturales (J ohnson \& Ali, 2004; Lui \& Suen, 2008; Humphreys, Johnson, Mason \&W hitehead, 2018), no existen estudiosque hayan analizado cuáles son los factores que podrían estar asociados con el rendimiento deportivo del equipo olímpico español en los JJ. 00. desde un enfoque multifactorial; ni que hayan anal izado las diferencias en función del sexo para estos modelos. Por ello, el objetivo de la presente investigación fue anal izar los factores socio-demográficos, económicos y deportivos relacionados con el éxito deportivo del equipo olímpico español en los JJ.00. de Pekín 2008, Londres 2012 y Rio de Janeiro 2016, exa minando si el sexo del deportista provoca variaciones en el modelo.

\section{Método}

\section{D iseño}

El diseño del presente estudio fue descriptivo y transversal. Tanto para el diseño como para el desarrollo del manuscrito se siguió la declaración de STROBE (Vandenbroucke et al., 2014).

Previo a la investigación se obtuvo la aprobación del comité de ética institucional de la U niversidad Católica de Murcia, España (código 19/ 6/ 2015).

\section{Participantes}

En la presente investigación se ha contado con un total de 875 deportistas pertenecientes al equipo olímpico español entre los años 2005 y 2016, de los cuales 499 eran hombres y 376 eran mujeres, suponiendo la muestra de la presente investigación el universo muestral. Estos deportistas participaron en los Juegos de Pekín 2008 ( $n=285)$, Londres $2012(n=281)$ y Rio de Janeiro $2016(n=309)$.

\section{Procedimiento}

Para la realización del presente estudio se analiza ron los siguientes datos

Parámetros socio-demográficos

Población: Se recogieron datos anuales sobre el total de habitantes, total de hombres y total de mujeres por año (Instituto Nacional de Estadística, 2016).

Índice de brecha de género: magnitud de la brecha existente entre mujeres y hombres en términos de salud (expectativa y proporción entre hombres y mujeres), educación (acceso a los diferentes niveles educativos), economía (salarios, participación y empleos altamente cualificados) y visibilidad (representación en estructuras de toma de decisión) (Foro Económico Mundial, 2016).

Índice de desarrollo humano: mide el progreso conseguido por un país en tres dimensiones: disfrutar de una vida larga y saludable, tener acceso a la educación y acceso a un nivel de vida digno (Programa de las N aciones U nidas para el Desarrollo, 2020).

Indice de paz global: mide el nivel de paz y la ausencia de violencia de un país a partir del análisis de variables internas (violencia y criminalidad) y externas (gasto militar y conflictos armados en los que participa el país) (Institute for Economics and Peace y el Centre for Peace 
and Conflict Studies, 2019).

Parámetros económicos

Producto Interior Bruto (PIB): indicador económico que refleja, cada año, el valor monetario de todos los bienes y servicios producidos por el país. Se utiliza para medir la riqueza de un país y se determina a partir del valor monetario de la producción de bienes y la exportación de servicios finales de un país durante un año (PIB = Consumo + Inversión + Gasto público - Importaciones) (International M onetary Fund, 2016).

Presupuesto deportivo: total de ingresos económicos aportados cada año por los organismos oficiales (Estado a través del CSD) y las Federaciones Nacionales (recursos Propios, Ayudas y A portaciones Públicas) (Consejo Superior de Deporte, 2016).

\section{Parámetros deportivos}

Licencias deportivas: total de licencias que, cada año, presentaban las Federaciones Nacionales de los deportes incluidos en los JJ.00. (Consejo Superior de Deporte, 2019).

Deportistas de alto nivel (DAN): deportistas de alto nivel y de alto rendimiento incluido en las resoluciones adoptadas, a tal efecto, por el de Secretario de Estado (Presidente del CSD), en colaboración con las federa ciones deportivas españolas y, en su caso, con las comunidades autónomas (CC.AA) (Consejo Superior de Deporte, 2016).

D eportistas del plan de la Asociación de D eportes O límpicos (ADO): D eportistas que cuentan con ayudas de laAsocia ción de Deportes cuyo principal objetivo es el apoyo, desar rollo y promoción de los deportistas nacionales de Alto Nivel para que puedan formar parte del equipo olímpico español en los JJ.00. (Asociación Española Deportista O límpicos, 2016).

D eportistas becados: total de deportistasque, cadatemporada, recibe una aportación económica, directa o indirecta, por algunos de los planes de apoyo al deporte de Alto Nivel que realiza alguna de las instituciones públicas (Consejo Superior de Deporte, 2016).

Índice de desarrollo deportivo: posición de cada país en el ámbito internacional en función de los resultados deportivos obtenidos cada año. Además de la puntuación global (IDD Global) se analizó la puntuación para la categoría masculina y femenina (Greatest Sporting Nation, 2016).

\section{Análisis de datos}

Para el tratamiento de las variables se utilizó el soft- ware estadístico IBM SPSS Statistic (Versión 24.0). Se realizó un análisis de componentes principales (ACP) para analizar las variables que podrían predecir la participación de los deportistas en los JJ.00. de manera general y en función del sexo. El ACP es un método algebraico/ estadístico multivariante que se utiliza para reducir, sintetizar y detectar la estructura subyacente en la información contenida en la matriz de datos (Abraira \& Pérez-deVargas, 1996; Almenara, González, García, \& Peña, 1998). Un problema clave en el análisis de datos multivariantes, es la reducción de la dimensionalidad a costa de una pequeña pérdida de información, razón por la que el ACP se convierte en una herramienta adecuada para lograr el objetivo de la presente investigación. Para comenzar, se determinó la calidad del análisis con el test Kaiser, Meyer y O Ikin (test KM O), para relacionar los coeficientes de correlación observados entre las variables, y el test de esfericidad de Bartlett, para evaluar la aplicabilidad del análisis factorial de las variables estudiadas. Al ser el val or de ambas pruebas significativo $(p<0.05)$ se procedió a realizar un análisis factorial, estableciéndose en la tabla de comunalidades el valor límite para validar el análisis en $>0.5$. Para explicar el peso de las variables según el criterio de la media aritmética con variables tipificadas, se seleccionaron aquell as componentes para las que sus raíces características o autovalores eran mayores que 1 (criterio de Kaiser). Por último, se analizaron diferentes modelos de componentes y la varianza explicada por los mismos, así como la correlación de factores dentro de ellos de manera global y en función del sexo.

\section{Resultados}

Las medidas de calidad del análisis resultaron ser moderadamente satisfactorias, con un índice de adecua ción muestral (Test KMO $=0,463$ ) y una prueba de esfericidad de Bartlett (Chi-cuadrado 164,6; gl = 55; $p<0,000)$. La tabla de comunalidades mostró que todas las variables estaban claramente representadas. Las variables con un nivel más elevado fueron el número de deportistas becados $(0,967)$, la población $(0,964)$, el presupuesto deportivo $(0,958)$ y el número de deportistas de alto nivel $(0,957)$. El valor de extracción más bajo lo tenía la variable perteneciente a los deportistas incluidos en el programaADO $(0,636)$.

El grado de representación que tiene cada uno de los componentes resultante, es decir, el grado de confiabilidad, para la población total, las mujeres y los 
hombres se muestra en la gráfica de sedimentación (Figura 1). Respecto al criterio de la media aritmética con variables tipificadas, este criterio se cumplió en los dos primeros componentesque, conjuntamente, explicaron el $79,81 \%$ de la varianza total. Las puntuacionestotales, varianza explicada por cada uno de los componentes y la matriz de variables que explicaban cada uno se muestran en laTabla 1. Con un $64,18 \%$ de la varianza expli-

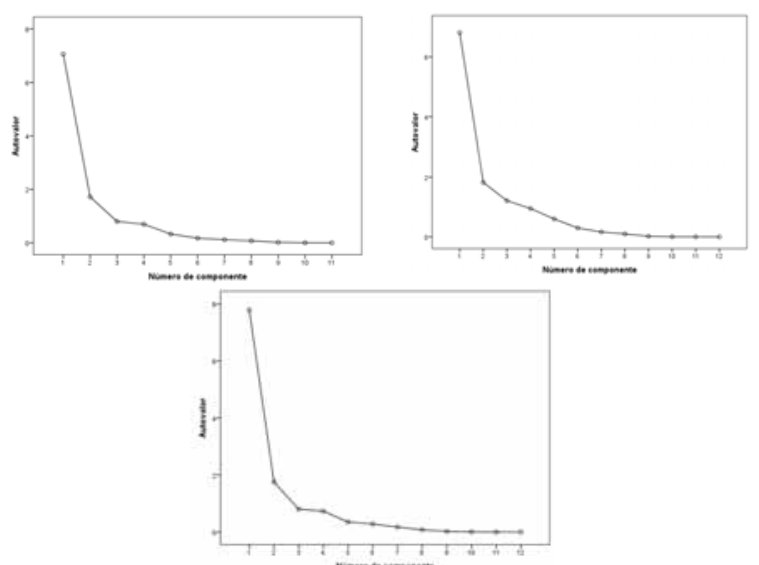

Figura 1. G ráfico de sedimentación resul tante del A nálisis de Componentes Principales Global para los deportistas del programa ol ímpico en general (izquierda), de las mujeres deportistas (centro) y los hombres deportistas (derecha).

Tabla 1.

Varianza explicada por cada uno de los componentes, puntuaciones totales y matriz de variables.

\begin{tabular}{|c|c|c|}
\hline Componente & Componente 1 & Componente 2 \\
\hline Población & 0,964 & 0,175 \\
\hline Índice de Brecha de Género & 0,106 & 0,756 \\
\hline Índice de D esarrollo Humano & 0,914 & $-0,083$ \\
\hline Índice de Paz G lobal & $-0,928$ & 0,034 \\
\hline Producto Interior Bruto (PIB) & 0,565 & 0,582 \\
\hline Presupuesto Deportivo & 0,958 & 0,163 \\
\hline Licencias D eportivas & 0,914 & 0,190 \\
\hline Deportistas deAlto Nivel (DAN) & 0,957 & $-0,038$ \\
\hline Deportistas del Plan ADO & $-0,636$ & 0,314 \\
\hline Deportistas Becados & 0,967 & 0,112 \\
\hline Índice de Desarrollo Deportivo & $-0,153$ & 0,817 \\
\hline Puntuación total & 7,060 & 1,720 \\
\hline Varianza explicada (\%) & 64,182 & 15,633 \\
\hline
\end{tabular}

Tabla 2.

Varianza explicada por cada uno de los compontes, puntuaciones totales y matriz de variables para las deportistas de sexo femenino del programa olímpico español.

\begin{tabular}{cccc}
\hline Componente & Componente 1 & Componente 2 & Componente \\
\hline Población & 0,957 & $-0,009$ & 0,149 \\
İndice de Brecha de Género & 0,101 & 0,663 & $-0,589$ \\
Índice de Desarrollo Humano & 0,920 & 0,147 & $-0,204$ \\
Índice de Paz Global & $-0,146$ & $-0,098$ & 0,932 \\
Producto Interior Bruto (PIB) & 0,571 & 0,304 & 0,430 \\
Presupuesto D eportivo & 0,951 & 0,131 & 0,131 \\
Licencias Deportivas & 0,921 & 0,088 & 0,095 \\
Deportistas deAlto Nivel (DAN) & 0,954 & $-0,151$ & $-0,070$ \\
Deportistas del Plan ADO & $-0,099$ & 0,876 & $-0,153$ \\
Deportistas Becados & 0,961 & $-0,040$ & $-0,087$ \\
Índice de Desarrollo Deportivo & $-0,910$ & $-0,182$ & 0,111 \\
Puntuación total & 6,820 & 1,830 & 1,212 \\
Varianza explicada (\%) & 56,831 & 15,213 & 10,104 \\
\hline
\end{tabular}

Tabla3.

Varianza explicada por cada uno de los compontes, puntuaciones totales y matriz de variables para las deportistas de sexo masculino del programa olímpico español.

\begin{tabular}{ccc} 
para las deportistas de sexo masculino del programa olimpico español. & \\
\hline Componente & Componente 1 & Componente 2 \\
\hline Población & 0,951 & 0,210 \\
Índice de Brecha de Género & 0,094 & 0,746 \\
İndice de Desarrollo Humano & 0,920 & $-0,061$ \\
İndice de Paz Global & $-0,177$ & 0,815 \\
Producto Interior Bruto (PIB) & 0,539 & 0,608 \\
Presupuesto D eportivo & 0,948 & 0,196 \\
Licencias Deportivas & 0,908 & 0,216 \\
Deportistas deAlto Nivel (DAN) & 0,947 & $-0,075$ \\
Deportistas del Plan ADO & $-0,636$ & 0,287 \\
Deportistas Becados & 0,957 & 0,147 \\
Inndice de Desarrollo Deportivo & $-0,947$ & $-0,947$ \\
Puntuación total & 7,801 & 1,750 \\
Varianza explicada (\%) & 65,00 & 14,61 \\
\hline
\end{tabular}

cada, el componente-1 agrupa población, presupuesto deportivo, licencias deportivas, deportistas de alto nivel (DAN) y deportistas becados. Respecto al componente-2, con unavarianza explicada del $15,63 \%$, el índice de brecha de género y el Producto Interior Bruto (PIB), son los que muestran un mayor coeficiente de correlación.

Cuando el análisis se realizó dividiendo la muestra en función de su sexo, se encontró que en el caso de las mujeres había tres componentes que cumplían con el criterio de la media aritmética con variables tipificadas, explicando en su conjunto un 82, 14\% de lavarianza. Las puntuaciones totales, varianza explicada por cada uno de los componentes y la matriz de variables que explicaban cada uno en el caso de las mujeres se encuentra en la tabla 2. Con un $56,83 \%$ de la varianza, el componente-1 estuvo compuesto por las mismas cinco varia bles que el modelo general más el índice de desarrollo humano. El componente-2, con un $15,21 \%$ delavarianza, quedó explicado por el número de deportistas incluidos en el programaADO y por el índice de la brecha de género. El componente-3, con un 10,10\% de lavarianza, incorpora como variable principal el índice de paz global.

A nal izando a los deportistas masculinos del progra maolímpico español (Tabla3), se encontraron dos componentes que cumplían con el criterio de la media aritmética con variables tipificadas, explicando en su conjunto un $71,61 \%$ de la varianza. Los mismos seis parámetros se vieron involucrados en el componente-1 (varianza explicada: 65,00\%). El índice de la brecha de género, el índice de paz global y el Producto Interior Bruto (PIB) fueron las claves que explicaron el Componente-2 (varianza explicada: 14,61\%).

\section{Discusión}

La presente investigación tuvo como objetivo analizar los factores socio-demográficos, económicos y deportivos relacionados con el éxito deportivo del equipo olímpico español en los tres últimos ciclos olímpicos, así como analizar las diferencias en función del sexo. LoS ACP realizados demostraron que España es un país que no muestra grandes desequilibrios entre hombres y mujeres en las variables que determinan la participación en los JJ.00. de sus deportistas. Así, se encontró un modelo muy similar en ambos sexos, relacionándose la participación en ambos sexos con los mismos parámetros que lo había hecho con el conjunto de la muestra. 
Cabe destacar que, si bien el modelo incluyó varia bles de todas las categorías, el número de deportistas becados fue el factor con más peso específico en el modelo. Esto podría deberse a la inversión realizada en deporte en las últimas décadas, sobre todo a raíz de la celebración de los J).00. de Barcelona 92 (Leiva \& Sánchez, 2019). En esta línea, en los últimos años una parte importante de estos recursos se han utilizado en ayudas para deportistas que han sido subvencionados a través del programaADO, los Centros de Alto Rendimiento (CAR) y otro tipo de ayudas. Durante la época analizada, el total de deportistas becados ha aumentado un $38.9 \%$ atendiendo a datos del Consejo Superior de Deportes. En base a los hallazgos del presente estudio, sería necesario seguir promocionando este tipo de acciones para aumentar las probabilidades de participa ción olímpica de los deportistas españoles.

0 tro factor con gran relevancia en base a los resultados fue la población. Esto podría deberse a que para que un sistema deportivo sea eficiente es necesario disponer de una masa crítica adecuada (población) de la cual puedan emerger un número suficiente de deportistas con las adecuadas capacidades y recursos para llegar representar a su país en las principales competiciones deportivas internacionales más relevantes (J).00. y Campeonatos del M undo) (Reyes, 2006). Consecuentemente, una mayor población aumenta las probabilida des de heterogeneidad del grupo y, por tanto, de que se encuentren sujetos con las características necesarias para rendir en modalidades deportivas diferentes (Rossing, Stentoft, Flattum \& Karbing, 2017). Si bien existen países con menor población y grandes resultados frutos de otros sistemas deportivos, tan dispares como el cubano, con el máximo aprovechamiento de capital humano en deportes seleccionados y gubernamentalmente priorizados (M oosa \& Smith, 2004).

No obstante, para que la población sea un factor relevante en la probabilidad de que un país tenga deportistas que vayan a los JJ.00., es necesario que la masa crítica que forma la misma practique deporte y le dé importancia a esta práctica, con el fin de incrementar la población potencial. A este respecto el número de licencias y el número de deportistas de alto nivel fueron otros de los factores destacados en el modelo. A finales del siglo XX se instauró en la población española una concienciación masiva sobre los beneficios de una vida activa (Solanellas \& Camps, 2017), lo que provocó un incremento del número de deportistas federados, especialmente en aquellos deportes incluidos dentro de los programas de los )J.0 0. hasta la actualidad (García
Ferrando, 2006; García-Ferrando \& Llopis, 2017). Estos movimientos fueron claves para establecer las bases de un proceso creciente en el tiempo que podría considerarse el embrión de los éxitos logrados por el deporte español en las últimas décadas (Cabello, Rivera, Trigueros, \& Pérez, 2011).

O tro hallazgo importante de la presente investigación es la importancia de los factores económicos sobre los índices de participación del equipo olímpico espa ñol. Al respecto, el presupuesto deportivo ha sido un factor que ha demostrado una gran relevancia. Durante el periodo del 2005 al 2016, la economía española sufrió una grave recesión, también experimentada por el resto de países desarrollados. A pesar de esta situación de recesión general, durante este periodo se intentó mejorar o mantener el nivel a costa de grandes esfuerzos de las diferentes instituciones involucradas en el mecenazgo del deporte (Grimal di-Puyana \& Sánchez-O liver, 2017). En base a los resultados de la presente investigación, la inversión realizada en deporte es un factor vital para la participación de los deportistas en los JJ.00. De esto se extra que es necesario disponer de recursos económicos suficientes para la promoción del deporte de alto rendimiento.

El índice de desarrollo humano fue un factor determinante tanto en el modelo general como en ambos sexos. Este índice analiza la esperanza de vida y la calidad de la misma. La posición de España ha estado habitualmente situada al rededor del puesto 25 a nivel mundial en este índice elaborado por la $\mathrm{ONU}$, si bien este índice decreció ligeramente durante el periodo analizado (U nited N ations D evelopment Programme, 2018), como consecuencia de la bajada del nivel de calidad de vida, puesto que la esperanza de vida se incrementó ligeramente durante este período (The World Bank, 2020). Los resultados del presente estudio concuerdan con estudios previos que han señalado la importancia del índice de desarrollo humano como factor determinante de la probabilidad de que un país tenga deportistas profesionales (Gomes-Sentone, Lopez-Gil, Caetano, $\&$ Cavichioll, 2019).

Los resultados de la presente investigación han evidenciado el índice de desarrollo deportivo, entendido como la posición de España en la clasificación internacional de la Greatest Sporting N ation (2016), no se contempló en la mayoría de los modelos presentados en la presente investigación. Esto podría deberse a que Espa ña suele mantenerse bastante estable en este ránking, situándose en el puesto 20 del mismo en general, el 17 para los hombres y el 22 para las mujeres. 
Un hallazgo sorprendente de la presente investiga ción fue que el PIB no fue un factor determinante en la probabilidad de participación de los deportistas españoles en los JJ.00. Estos resultados contradicen los encontrados por estudios previos, en los que se halló que existe una correlación directa entre el PIB de un país y el número de participantesy medal las obtenidas en unos JJ.00., tras analizar los datos de 20 naciones de diferente espectro macroeconómico. Las divergencias con España podrían deberse a que, a pesar de que su PIB se sitúa en el top15 mundial en los años analizados, siendo el ranking bastante estable (International M onetary Fund, 2019), sólo un 0,01\% del mismo seha destinado a deporte (M inisterio de Cultura y Deporte, 2020), Io que hace que la inversión en este ámbito sea insuficiente como para obtener rédito de la misma. Sin embargo, en base a los resultados del resto de variables económicas, el dinero que se invierte en deporte está siendo fructífero.

El plan ADO parece no tener un gran peso en la participación en los JJ.00. en el caso de los hombres, pero sí en el de las mujeres. Estudios previos han apuntado queal comparar el éxito en los JJ.0 0. de los becados con el programa ADO, las deportistas obtienen mejores resultados que los hombres (Leiva-Arcas, SánchezPato \& Martínez-Patiño, 2020). Esto podría deberse al menor nivel de profesionalización que hay entre ellas, lo que les hace más difícil poder dedicarse exclusiva mente al deporte (López, 2017), cuestión que sí que es promovida por este programa, lo que facilitaría que aquellas que se benefician de esta subvención se puedan dedicar profesionalmente al deporte, aumentando sus posibilidades de éxito. En base a esto, podría ser más rentable obtener mejores resultados invirtiendo en deporte femenino, tal y como demuestran investiga ciones previas (Leiva-Arcas et al., 2020). El hecho de que no se encontrara esta relación en los hombres, a pesar de que sí se halló un vínculo entre la participación olímpica y otras becas, podría deberse a que el porcentaje de deportistas que disfrutan del plan ADO es muy limitado al compararlo con otros programas de ayuda al deportista (Leiva-Arcas et al., 2020).

La brecha de género mostró ser relevante tanto para el modelo general como en función del sexo. Por lo tanto, se encuentran diferencias en términos de salud, educación, economía y visibilidad en función del sexo. Esto es una realidad aún lejos de poder ser solucionada, aunque las diferencias se han reducido progresivamente como demuestran los datos (World Economic Forum, 2020). El esfuerzo de la mujer española por ocupar su puesto en la sociedad es un hecho incuestionable y, en esa lucha, las deportistas españolas han hecho una aportación clave en sus reivindicaciones (Leruite, M artos \& Zabala, 2015). A poyándose en las disposiciones de la Carta de las Naciones U nidas, la Declaración U niversal de los Derechos Humanos, y la Convención de las $\mathrm{Na}$ ciones U nidas sobre la Eliminación de Todas Formas de Discriminación Contra las Mujeres, se ha tratado de atajar los clásicos estereotipos discriminatorios que ca racterizaron las primeras etapas del deporte moderno (Ramírez-M acías \& Piedra de la Cuadra, 2011). Sin embargo, a pesar de estos esfuerzos es un hecho que el deporte sigue siendo predominantemente masculino. Es un hecho que la incorporación femenina a determinadas disciplinas y ámbitos deportivos ha sido lenta y escalonaday no exenta de barreras de índole social, económica y cultural (Isorna, Felpeto, Alonso, Gómez, \& Rial, 2019). No en vano, un $76.2 \%$ de los deportistas federados españoles son hombres (Consejo Superior de Deportes, 2019). Aunque la visibilidad del deporte femenino ha aumentado, especial mente en los medios de comunicación, su presencia sigue siendo testimonial y altamente estereotipada (Ruíz-Rabadán \& M oya-M ata, 2020). Por tanto, siguen siendo necesario programasque promuevan la igualdad de acceso a la práctica del deporte independientemente del sexo del deportista.

El índice de paz global también resultó ser significa tivo para el modelo, al dividir a la muestra en función del sexo. En las últimas décadas, España ha sufrido una evolución de este indicador como consecuencia del abandono de la lucha armada por parte de la banda terrorista ETA y por la ilusión de vivir en una sociedad plena mente democrática integrada en la U nión Europea, dentro de un nuevo orden internacional. Esto podría promover la práctica de deporte, aumentando las probabilidades de conseguir el éxito deportivo (Darnell, 2012).

\section{Conclusiones}

En conclusión, aunque el análisis del presente estudio se realiza en un periodo en el que el deporte espa ñol ha alcanzado excelentes logros internacionales, la participación de los deportistas españoles en los JJ. 00 . se debe a una gran amal gama de variables socio-demográficas, económicas y deportivas. A la luz de los resultados obtenidos, todo parece indicar que, pese a ser el sistema deportivo actual aún eficiente, es necesario rea lizar un replanteamiento desde un punto de vista global para ajustar el modelo a las necesidades del deporte 
actual de máximo rendimiento, teniendo en cuenta la limitación depoblación con laquecuentaEspañaen comparación con otros países. Si bien el patrón es bastante similar en ambos sexos, de la presente investigación se puede extraer que el modelo sigue siendo eficaz sobre todo en el caso del deporte femenino. Respecto a las futuras líneas de investigación, se debería realizar un estudio pormenorizado de las alternativas futuras donde con un aceptable coste/ beneficio, se logre al canzar mejores resultados de los hasta ahora conseguidos.

\section{Agradecimientos}

Los autores agradecen al Comité 0 límpico Español por la cesión de los datos para este estudio y al Dr. Juan García Manso por su inestimable ayuda para la realiza ción de este trabajo.

\section{Referencias}

Abraira,V. \& Pérez-deVargas, A. (1996). M étodosmultivariantes en bioestadística. Madrid: Editorial Centro de Estudios Ramón Areces.

Almenara, J., González, J. L., García, C., \& Peña, P. (1998). ¿Q uéesel análisisde componentesprincipales?, Jano, 1268, 58-60.

Asociación Española Deportista O límpicos (2016). Listado deportistas. Madrid: Asociación EspañolaD eportistaO límpicos. Disponible en: https: / / www. coe.es/ coe/ bd_perso. nsf / aedoinicio? 0 penFor $m \&$ Count $=99$ $9 \&$ ResortAscending $=4 \&$ Seq $=8$

Cabello, D., Rivera, E., Trigueros, C., \& Pérez, I. (2011). A ná lisis del modelo del deporte federado español del siglo XXI. Revista Internacional de Medicina y Ciencias de la Actividad Física y el Deporte, 11 (44), 690-707.

Consejo Superior de Deporte (2016). Consejo Superior de De porte. Madrid: M inisterio de Culturay D eporte. Disponible en: https:/ / www.csd. gob.es/ es

Consejo Superior de Deportes (2019). Licencias federadas se gun sexo por federacion. 2019. Madrid: Ministerio de Cultura y Deporte. Disponible en: https:// ww w. culturaydeporte. gob.es/ dam/ jcr:4b8664861c11-4dc4-a528-8611c431a7e7/ licencias-federadaspor-sexo.pdf

Curi, M., Knijnik, J., \& Mascarenhas, G. (2011). The Pan American Games in Rio de Janeiro 2007: Consequences of a sport megarevent on a BRIC country. International Review for the Sociology of Sport, 46 (2), 140-156. doi: $10.1177 / 1012690210388461$

Darnell, S. (2012). Sport for development and peace: A critical sociology. London: A\&C Black.

De Bosscher, V., DeKnop, P., van Bottenburg, M., Shibli, S. \& Bingham, J. (2009). Explaining international sporting success: A n international comparison of elite sport systems and policies in six countries. Sport Management Review, 12 (3), 113-136. doi: 10.1016/ j.smr.2009.01.001

Foro Económico M undial (2016). Salario anual medio, mediano y modal. Salario por hora. Brecha salarial de género (no ajustada) en salarios por hora. Madrid: Instituto Nacional de Estadística. Recuperado de: https: / / www.ine.es/ ss/ SatelliteX =es_ES\& $=$ INESeccion_C\&cid=1259925408327\& $p=1254735110672 \&$ pagename=Productos/Servicios\%2FPYSLajout

Garcia, B. \& Miah, A. (2012). The Olympics: The Basics. Londres: Routledge.

GarcíaFerrando, M. (2006). Veinticinco años de análisis del comportamiento deportivo de la población española (1980-2005). Revista Internacional de Sociología, 64(44), 15-38.

GarcíaFerrando, M. \& LlopisGoig, R. (2017). La popularización del deporteen España: encuestas dehábitos deportivos 1980 2015. Madrid: CIS-Centro de Investigaciones Sociológicas.

Greatest Sporting Nation (2016). The Quest for the best. Londres: G reatest Sporting N ation. Recuperado de: https: / / greatestsportingnation. com/ country/ spain

Green, M. (2007). Olympic glory or grassroots development?: Sport policy priorities inAustralia, Canada and the United Kingdom, 1960-2006. The International Journal of theH istory of Sport, 24 (7), 921-953. doi: 10.1080/ 09523360701311810.

Gomes-Sentone, R., Lopez-Gil, J. F., Caetano, C. I., \& Cavichioll, F. R. (2019). Relationship between human development index and the sport results of Brazilian swimming athletes. Educación Física y Deportiva, 14 (proc5), S2009-S2018. doi: 10.14198/ jhse.2019.14.Proc5.22

Grimaldi-Puyana, M . \& Sánchez-O liver, A. J. (2017). Tamaño y evolución económica de los clubes de baloncesto SAD después de la crisis económica de 2008. Cuadernos de Psicología del Deporte, 17(3), 137-142.

Haake, S. J. (2009). The impact of technology on sporting performance in 0 lympic sports. Journal of Sports Sciences, 27(13), 1421-1431. doi:10.1080/ 02640410903062019.

H offmann, R., Ging, C., \& Ramasamy, B. (2002). The SocioEconomic Determinants of International Soccer Performance. Journal of Applied Economics, 5, 253-272.

Humphreys, B. R., Johnson, B. K., Mason, D. S., \&W hitehead, J. C. (2018). Estimating the value of medal success in the Olympic Games. Journal of Sports Economics, 19(3), 398416. doi:10.1177/ 1527002515626221.

International M onetary Fund (2016). Country data. Washington: International M onetary Fund. Recuperado de: https:/ / www. imf. org/ en/ Countries/ ESP\# countrydata

International M onetary Fund (2019). GDP, current prices. Washington: International M onetary Fund. Recuperado de: https: / / ww w. imf. org/ external/ datamapper/ NGDPD@WEO/OEMDC/ ADVEC/WEOWORLD 
Institute for Economics and Peace y el Centre for Peace and Conflict Studies (2019). Global Peace Index 2019. Sidney: Institute for Economics \& Peace.

Instituto Nacional de Estadística (2016). Cifras de Población. M adrid: Instituto Nacional deEstadística. Recuperado de: https: / / www.ine.es/ dyngs/ I N Ebase/ es/ operacion. htm?c=Estadistica_ C\&cid $=1254736176951$ \&menu $=$ ultiD atos\&idp $=1254735572981$

Isorna Folgar, M., Lamas, M., Alonso Fernández, D., Gómez Salgado, P., \& Rial Boubeta, A. (2018). Mujer y piragua: estudio de las variables moduladoras del abandono de portivo de las mujeres piragüistas en modalidades olímpicas. Retos, (35), 320-325. https:/ / doi.org/ 10.47197/ retos. v0i35.66800

Johnson, D. K. N. \& Ali, A. (2004). A tale of two seasons: participation and medal countsat the Summer andW inter O lympic Games. Social Science Quarterly, 85 (4), 974-993. doi:10.1111/ j.0038-4941.2004.00254.x

Leiva, A. \& Sánchez, A. (2019). A nál isis de los resultados de España en su participación en los Juegos 0 límpicos de verano. En A. Aragón y J. Pernas. (Eds.). El olimpismo en España. Una mirada histórica de sus orígenes a la actualidad (pp. 295-336). Barcelona: Fundación O límpica Barcelona.

Leiva-Arcas, A., Sanchez-Pato, A. \& M artinez-Patino, M. J. (2020). Impact Analysis of Ado Plan in the Spanish O lympic Results. Revista Internacional de Medicina y Ciencias de la Actividad Fisica y el Deporte, in press.

Leruite, M. T., M artos, P., \& Zabala, M. (2015). Análisis del deporte femenino español de competición desde la perspectiva de protagonistas clave. Retos: nuevas tendencias en educación física, deporte y recreación, (28), 3-8.

López, M. J. (2017). Mujer, discriminación y deporte. Madrid: Editorial Reus.

Lui, H. K., \& Suen, W. (2008). Men, money, and medals: An econometric analysis of the Olympic Games. Pacific Economic Review, 13(1), 1-16. doi:10.1111/ j.14680106.2007.00386.x

Ministerio de Cultura y Deporte (2020). Anuario de Estadísticas Deportivas. Gasto público vinculado al deporte. Madrid: MCUD. Recuperado de: https:// www. culturaydeporte. gob.es/ dam/ jcr:7f3cba99-6eal4420-9b96-5e54e1459bd8/ nota-resumen-gastopublio-vinculado-al-deporte. pdf

Moosa, I.A., \& Smith, L. (2004). Economic development indicators as determinants of medal winning at the Sydeny O lympics: an extremeboundsanalysis. Australian Economic Papers, 43(3), 288-301.

M oscoso, D., Fernández, J., \& Rodríguez, A. (2014). De la democratización del deporte a la hegemonía de los mercados: El caso español. M ovimento, 20, 109-124.

Perrino Peña, M. \& Vicente Pedraz, M. (2018). O limpismo en la revista Citius, Altius, Fortius (1959-1976): Ios inicios de la crítica al M ovimiento 0 límpico en España. Re tos, (34), 177-182. https:// doi.org/ 10.47197/ retos. v0i34.59959

Programa de las N aciones U nidas para el D esarrollo (2020). Panorama general. Informe sobredesarroll lo humano 2019. N ueva York: Naciones U nidas para el D esarrollo.

Ramírez-Macías, G. \& Piedrade la Cuadra, J. (2011). A nálisis de la obra de José M aría Cagigal en relación con el concepto de mujer y su inclusión en el deporte. Apunts. Educación Física y Deportes, 3 (105), 66-72. doi:10.5672/ apunts. 2014-0983.es. (2011/ 3). 105.0

Reyes, M. A. (2006). Política deportiva: factores reales del sistema deportivo. Liberabit, 12 (12), 87-94.

Rossing, N. N., Stentoft, D., Flattum, A., \& Karbing, D. S. (2017). Influence of population size, density, and proximity to talent clubs on the likelihood of becoming elite youth athlete. Scandinavian Journal of M edicine \& Science in Sports, 28 (3), 1304-1313. doi: 10.1111/ sms. 13009

Ruiz Rabadán, S. \& M oya-M ata, I. (2020). Las deportistas olímpicas en los libros de texto de educación física: ipresencia 0 ausencia de referentes en nuestro alumnado?. Retos, 38(38), 229-234. https:/ / doi.org/ 10.47197/ retos. V38i38. 74833

Sánchez, A., Isidori, E. , Arias, J. L., \& Bada, J. D. D. (2018). Modelo de carrera dual universitario. El caso de los deportistasestudiantes. Cizur M enor: Aranzadi.

Silva, D., Ribeiro, O., Silvestre, B., \& Salerno, M. (2020). Copa Mundial de la FIFA y Juegos O límpicos y Paralímpicosen Brasil: legadosen laciudad de CampinasSP. Retos, (40), 86-94. https:/ / doi.org/ 10.47197/ retos. v1i40.82617

Solanellas, F. \& Camps, A. (2017). Los Juegos 0 límpicos de Barcelona, 25 años después (1). Apunts. Educación Física y Deportes, 127, 7-26. doi:10.5672/ apunts. 20140983.es. (2017/ 1). 127.01

TheWorld Bank (2020). Life expectancy at birth, total (years) Spain. Washington: World Bank Group. Disponible en: https:/ / data. worldbank.org/ about/ contact

United Nations Development Programme (2018). Human Development Index trends, 1990-2018. New York: Human Development Reports. Disponible en: http:// hdr.undp.org/ en/ content/ table-2-humandevelopment-index-trends-1990-2018

Vandenbroucke, J. P., Von Elm, E., Altman, D. G., Gøtzsche, P. C., Mulrow, C. D., Pocock, S. J., ... , Initiative, S. (2014). Strengthening the Reporting of 0 bservational Studies in Epidemiology (STRO BE): explanation and elaboration. International Journal of Surgery, 12 (12), 1500-1524. doi: 10.1371 / journal. pmed. 0040297

World Economic Forum (2020). Insight Report. Global Gender Gap Report 2020. Cologny: World Economic Forum. 